\title{
TEMPERATURE MEASUREMENT OF CRYOGENIC NITROGEN JETS AT SUPERCRITICAL PRESSURE
}

\author{
H. Tani ${ }^{1}$, S. Teramoto ${ }^{2}$, T. Toki ${ }^{2}$, S. Yoshida ${ }^{2}$, \\ K. Yamaguchi ${ }^{2}$, and K. Okamoto ${ }^{2}$ \\ ${ }^{1}$ Japan Aerospace Exploration Agency (JAXA) \\ Tsukuba, Ibaraki 305-8505, Japan \\ ${ }^{2}$ University of Tokyo, Department of Aeronautics and Astronautics \\ Hongo, Tokyo 113-8656, Japan
}

\begin{abstract}
The temperatures of transcritical and supercritical nitrogen jets were measured to explore the influence of "pseudovaporization" upon cryogenic propellant mixing in high-pressure rocket chambers. Pseudovaporization is the large thermodynamic transition near the pseudocritical temperature under transcritical conditions, which can include a drastic density change and large peak of isobaric specific heat. A decline in the rise of temperature along the jet centerline of the transcritical jet was caused at the position where the local temperature reached nearpseudocritical temperature. This can be considered to be due to the large peak of isobaric specific heat. The density jump appeared near the pseudocritical temperature, which can be correlated to the sudden expansion due to pseudovaporization. The axial profiles of the temperature and density of the supercritical jet monotonically increased and decreased, respectively, in the downstream region of the end of the jet potential core. Similar to the axial profiles, the radial profiles of the temperature were influenced by the pseudovaporization - i. e., the temperature rise in the radial direction became very shallow in the region where the local temperature was still lower than the pseudocritical temperature. The full width at half maximum of the density profiles stayed almost constant further downstream of the end of the jet potential core, whereas that of the mass fraction profiles of the incompressible variable-density jet began to increase near the end of the potential core. Hence, the evolutions of jet mixing layers of transcritical jets and variable-density jets can be considered to differ due to pseudovaporization.
\end{abstract}

\section{NOMENCLATURE}

\section{Symbols}

$D$ inner diameter of injector, $2 \mathrm{~mm}$

$h$ specific enthalpy 
$L \quad$ full width at half maximum

$P$ pressure

Re Reynolds number based on $D, \operatorname{Re}=\rho V D / \mu$

$T$ temperature

$V \quad$ injection velocity

$x, r$ coordinates

$\mu \quad$ viscosity

$\rho$ density

\section{Subscript and superscripts}

amb ambient fluid

inj injection fluid

+ normalization by injection and ambient fluids, $\rho^{+}=\left(\rho-\rho_{\text {amb }}\right) /\left(\rho_{\text {inj }}\right.$ $\left.-\rho_{\mathrm{amb}}\right)$

\section{INTRODUCTION}

Propellant mixing phenomena in liquid rocket combustors are important in the sense that they have a significant impact on the combustor performance. In particular, most liquid rocket engines used in the first stage of launch vehicles operate at a pressure above the critical pressure of oxygen. Hence, understanding of the mixing processes involved in cryogenic jets under supercritical pressures is necessary.

Figure 1 illustrates the thermodynamic phase diagram of oxygen using pressure and temperature. The arrow in Fig. 1 schematically shows the thermodynamic change of oxygen in such high-pressure rocket combustors. Cryogenic oxygen at an initially subcritical temperature is injected into an environment that exceeds the critical pressure and temperature (5.04 MPa and $154.6 \mathrm{~K}$, respectively). The thermodynamic change from subcritical temperature to supercritical temperature under supercritical pressures is called "transcritical" [1]. The pseudocritical temperatures under transcritical conditions correspond to the boiling temperatures at subcritical pressures. At pseudocritical temperatures, oxygen experiences a large thermodynamic transition from liquid-like properties to gas-like properties, such as a large density change and considerable amount of expansion heat. This large thermodynamic transition is referred to "pseudovaporization" or "pseudoboiling" [2]. Most studies on fluid mixing under supercritical pressures considered often only the gas-like properties of supercritical fluids, such as the diminishment of gas-liquid surface tensions; however, the fact that pseudovaporization is involved in fluid mixing phenomena is also important.

Several numerical studies on transcritical jets have been reported in the last decade [3-6]. Hosangadi et al. [4] simulated cryogenic nitrogen/gaseous nitrogen coaxial jets under transcritical conditions by means of a hybrid large-eddy sim- 


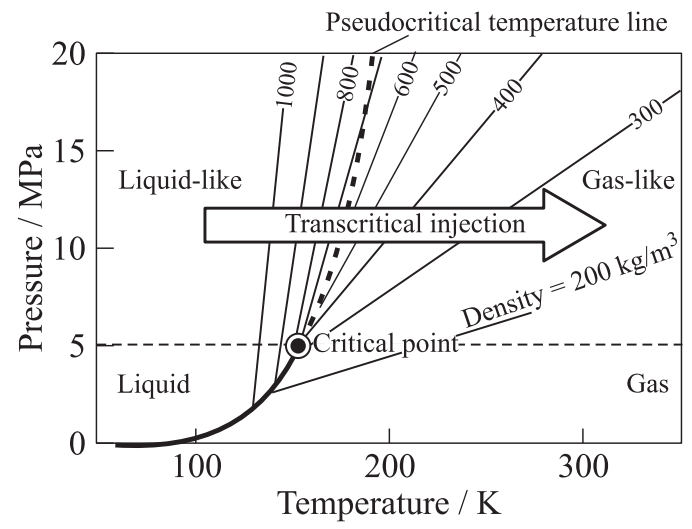

Figure 1 Schematic of thermodynamic state of oxygen in liquid rocket combustors

ulation (LES) / Reynolds-averaged Navier-Stokes (RANS) simulation method. They suggested that pseudovaporization influences the unsteady behavior of the cryogenic jet core. Zong and Yang [3] and Jarczyk and Pfitzner [6] conducted LES of cryogenic nitrogen jets under transcritical conditions. They showed that strong anisotropy of turbulence occurs in the initial mixing region owing to the large density stratification between the injected and ambient fluids.

Quantitative measurement data of transcritical jets are indispensable for both validation of these numerical studies and better understanding of mixing phenomena. However, only a few sets of quantitative experimental data have been made public compared with data on incompressible variable-density jets [7]. To the best of the authors' knowledge, Raman-scattering-based density profiles of transcritical nitrogen jets are the only quantitative data that have been published $[1,8]$. These profiles showed that the spatial growth rate of transcritical jets agrees with those predicted by the theoretical equations and measurements for incompressible variable-density turbulent jets. However, the jet temperature at the injector exit almost reaches the pseudocritical temperature; so, pseudovaporization may only influence the region close to the injector. In addition, the error of the Raman scattering measurement in high-pressure environments becomes large because scattering due to the high-density ambient fluids weakens the signals of the jets [9].

The purpose of this study was to explore the influence of pseudovaporization upon jet mixing by using quantitative data on transcritical jets. High-pressure injection tests were conducted and temperature profiles of a transcritical nitrogen jet were obtained by means of a temperature sensor traverse. For comparison, temperature profiles of a supercritical nitrogen jet at a temperature over the pseudocritical temperature were also measured. 


\section{EXPERIMENTAL SETUP AND CONDITIONS}

\subsection{Experimental Setup}

In the experiment, cryogenic nitrogen was employed as an injectant because nitrogen is thermodynamically similar to oxygen. The critical pressure, temperature, and density of nitrogen are $3.4 \mathrm{MPa}, 126.2 \mathrm{~K}$, and $313.3 \mathrm{~kg} / \mathrm{m}^{3}$, respectively.

Figure 2 shows a schematic diagram of the experimental setup. The test stand comprises an injector, high-pressure chamber, and supply system for the cryogenic nitrogen. Cryogenic nitrogen is first pressurized to the required level by high-pressure nitrogen gas in an $\mathrm{LN}_{2}$ pressurized reservoir and then injected at a cryogenic temperature into the high-pressure chamber. The high-pressure chamber is filled with nitrogen gas at a supercritical pressure and ambient temperature. The injector is placed in the top flange of the high-pressure chamber. Figure 3 shows the injector details. The injector has an outer nozzle to blow a coflow jet. This paper only presents the results of a round jet from the inner nozzle. The injector is about half the size of practical rocket engine injectors.

The temperature of the cryogenic nitrogen is controlled using an electronic heater installed in the reservoir as shown in Fig. $2 b$. The injector is not insulated,

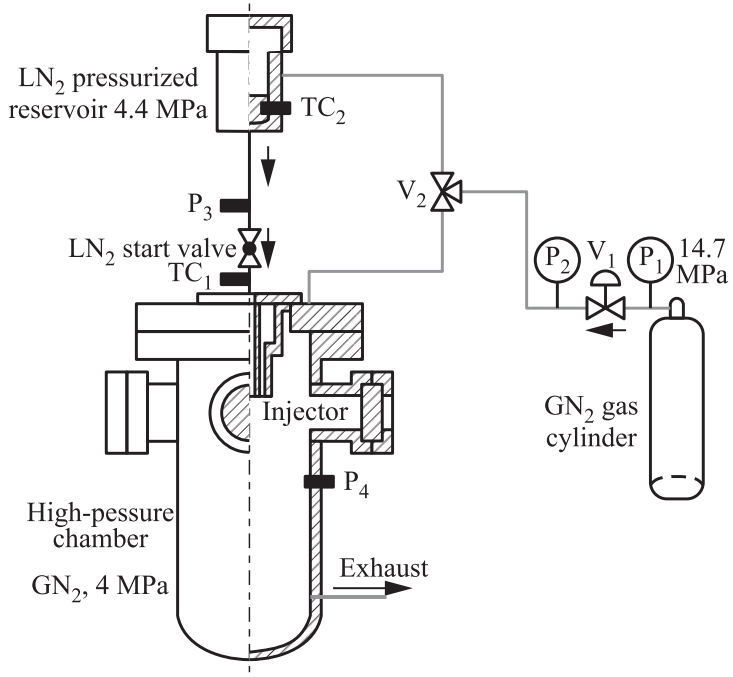

(a)

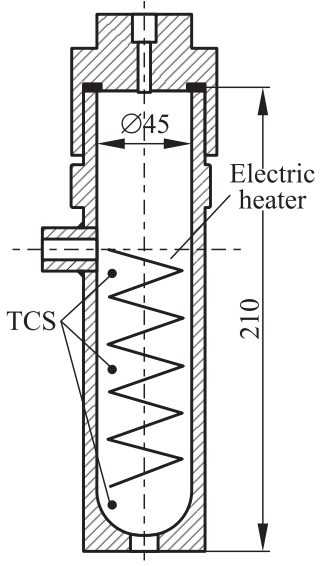

(b)

Figure 2 Simplified diagram of experimental setup (TC - thermocouple; P pressure sensor; and $\mathrm{V}$ - valve): (a) flow diagram and (b) $\mathrm{LN}_{2}$ pressurized reservoir and electric heater (dimensions are in millimeters) 

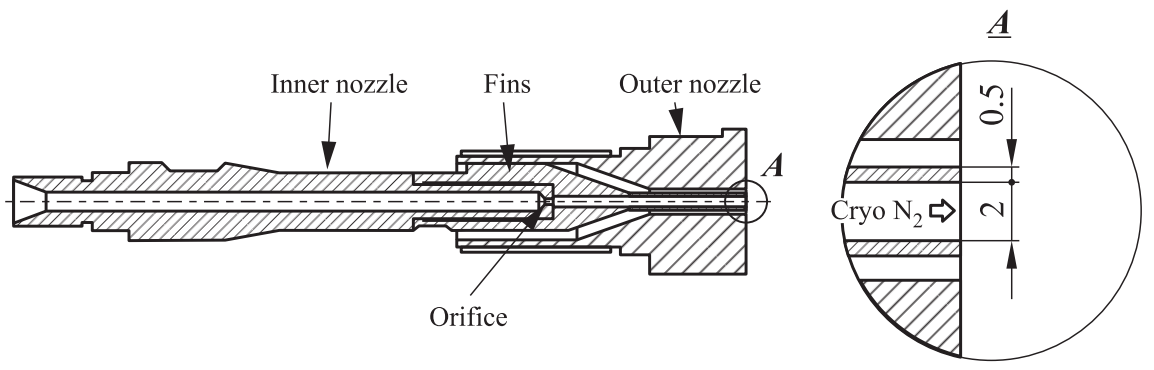

Figure 3 Schematic of injector assembly. Dimensions are in millimeters

but the injector is carefully cooled prior to injection so that the temperature of the nitrogen at the injector exit is maintained within $\pm 0.9 \mathrm{~K}$ of the target value.

\subsection{Test Conditions}

The temperature profiles of cryogenic nitrogen jets were measured under two different flow conditions: transcritical and supercritical. Table 1 summarizes the flow conditions. The ambient pressure of 4.0 MPa corresponds to 1.18 times of the critical pressure of nitrogen. The pseudcritical temperature at this pressure is approximately $129.7 \mathrm{~K}$. The present jet temperature of the transcritical case was about $27 \mathrm{~K}$ below the pseudocritical temperature; so, the influence of pseudovaporization would be observed downstream of the jet core, not only in the region close to the injector exit.

Figure 4 shows the density, specific enthalpy, and isobaric specific heat of nitrogen at 4.0 MPa. The pseudocritical temperature exists between the injection temperature of cryogenic nitrogen and the ambient gaseous nitrogen in the transcritical case. In the vicinity of the pseudocritical temperature, density greatly

Table 1 Injection conditions

\begin{tabular}{llcc}
\hline \multicolumn{2}{c}{ Parameter } & Transcritical jet & Supercritical jet \\
\hline \multirow{4}{*}{ Chamber } & $P_{\mathrm{amb}}, \mathrm{MPa}$ & \multicolumn{2}{c}{$4.0( \pm 0.02)$} \\
& $T_{\mathrm{amb}}, \mathrm{K}$ & \multicolumn{2}{c}{$293( \pm 3)$} \\
& $\rho_{\mathrm{amb}}, \mathrm{kg} / \mathrm{m}^{3}$ & \multicolumn{2}{c}{$46.3( \pm 0.3)$} \\
& $h_{\mathrm{amb}}, \mathrm{kJ} / \mathrm{kg}$ & \multicolumn{2}{c}{$295( \pm 4)$} \\
\hline \multirow{4}{*}{ Injection } & $T_{\mathrm{inj}}, \mathrm{K}$ & $103.5( \pm 0.9)$ & $138( \pm 2)$ \\
& $\rho_{\mathrm{inj}}, \mathrm{kg} / \mathrm{m}^{3}$ & $690.8( \pm 5)$ & $160.3( \pm 10)$ \\
& $h_{\mathrm{inj}}, \mathrm{kJ} / \mathrm{kg}$ & $-64.8( \pm 2)$ & $88.4( \pm 6)$ \\
& $V_{\mathrm{inj}}, \mathrm{m} / \mathrm{s}$ & 7.9 & 16.4 \\
& $\mathrm{Re}$ & $1.4 \cdot 10^{5}$ & $4.1 \cdot 10^{5}$ \\
\hline Density ratio $\rho_{\mathrm{inj}} / \rho_{\mathrm{amb}}$ & 15 & 3.5 \\
\hline
\end{tabular}




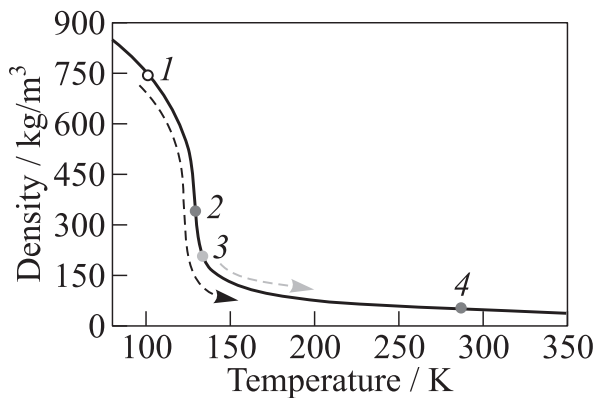

(a)

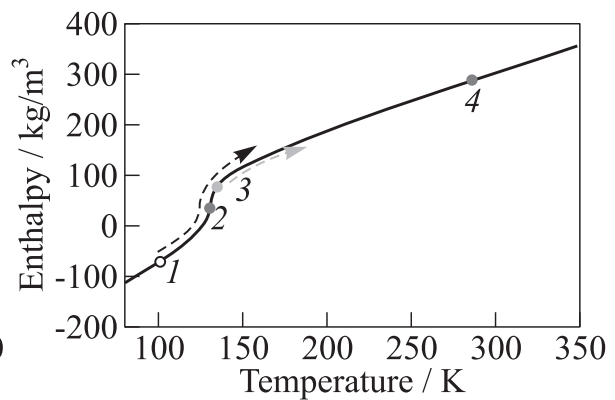

(b)

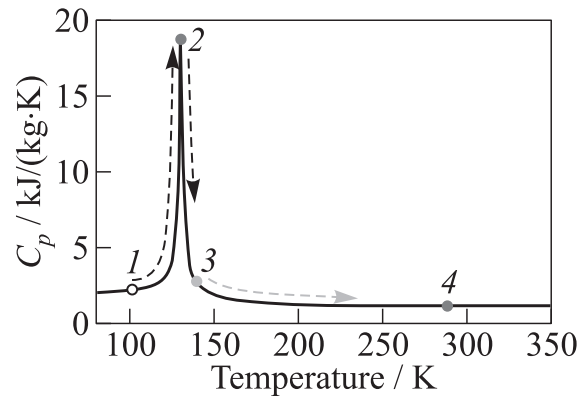

(c)

Figure 4 Thermodynamic properties of nitrogen at $4.0 \mathrm{MPa}$ : $(a)$ density; $(b)$ specific enthalpy; and $(c)$ isobaric specific heat. Characteristic temperatures: 1 - transcritical injection $(103.5 \mathrm{~K}) ; 2$ - pseudocritical temperature $(129.7 \mathrm{~K}) ; 3$ - supercritical injection $(138 \mathrm{~K})$; and 4 - chamber $(293 \mathrm{~K})$

changes and there is a significant change in the specific enthalpy. This large enthalpy change is similar to the latent heat of vaporization at subcritical pressures. Hence, a large peak of isobaric specific heat appears at the pseudocritical temperature. On the other hand, the supercritical jet does not experience this large thermodynamic transition.

\subsection{Measurement}

Time-averaged backlit images were obtained to compare the flow field features of the transcritical and supercritical jets.

A temperature sensor probe was mounted on a two-axis traversing device installed downstream of the injector as shown in Fig. 5. This figure also describes the coordinates for the temperature measurement. For the transcritical jet, the axial temperature profile was measured by a NETSUSHIN 0.5-millimeterdiameter platinum resistance temperature sensor; the radial temperature profile, 
where spatial resolution is more important, was measured with a 0.25-millimeter-diameter K-type thermocouple. For the supercritical jet, the axial and radial temperature profiles were measured with a 0.25-millimeter-diameter K-type thermocouple.

The temperature sensors were calibrated against a high-accuracy Cernox sensor and the measurement accuracy of the platinum resistance sensor was evaluated to be $\pm 0.3 \mathrm{~K}$. The uncertainty for the thermocouple was much larger but has not been quantified at present. The flow rate of the cryogenic nitrogen was measured at an orifice inserted just upstream of the run valve.

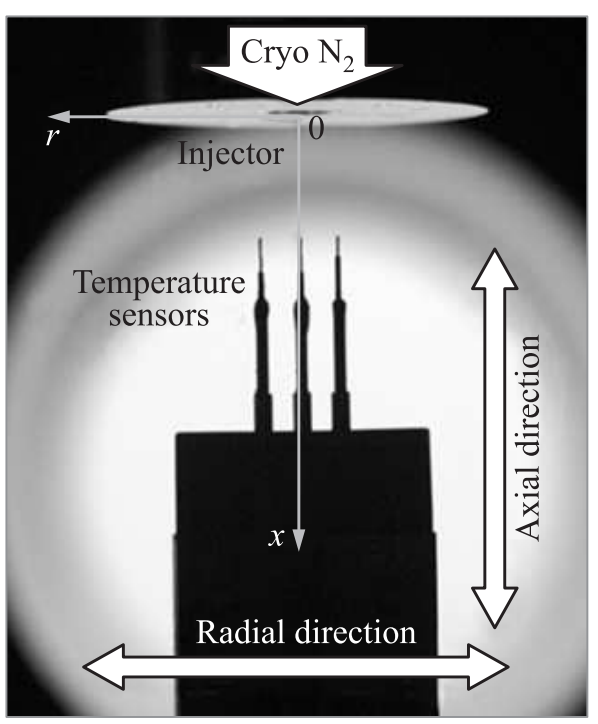

Figure 5 Temperature measurement probe

\section{RESULTS AND DISCUSSION}

\subsection{Visualization}

Figure 6 illustrates the time-averaged backlit pictures of the transcritical and supercritical jets. A dark core region of the transcritical jet was clearly observed and had a length of about $20 \mathrm{D}$ from the injector exit. In contrast, the shadow of the supercritical jet seemed fainter and shorter than the dark core of the transcritical jet.

\subsection{Axial Profiles}

Figure 7 shows the axial profiles and axial gradients of the temperature along the jet centerline. The temperature profile of the transcritical jet can be divided into four regions:

(I) a jet potential core region with almost constant temperature $(0<x / D$ $<5)$;

(II) linear increase at $5<x / D<15$;

(III) very shallow increase near $x / D=15$; and

(IV) linear increase again with a larger gradient at $x / D>20$. 


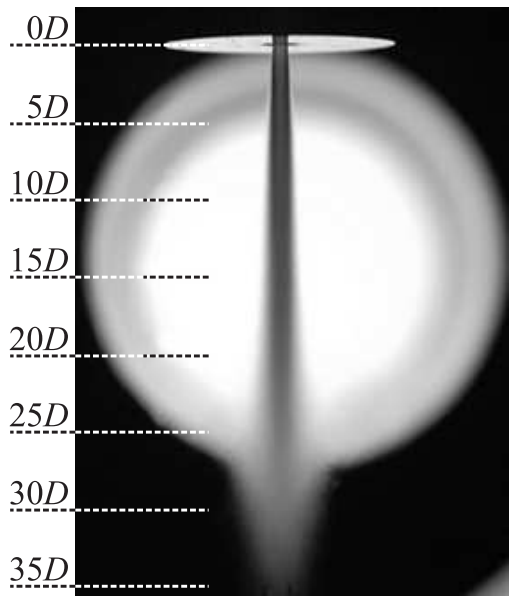

(a)

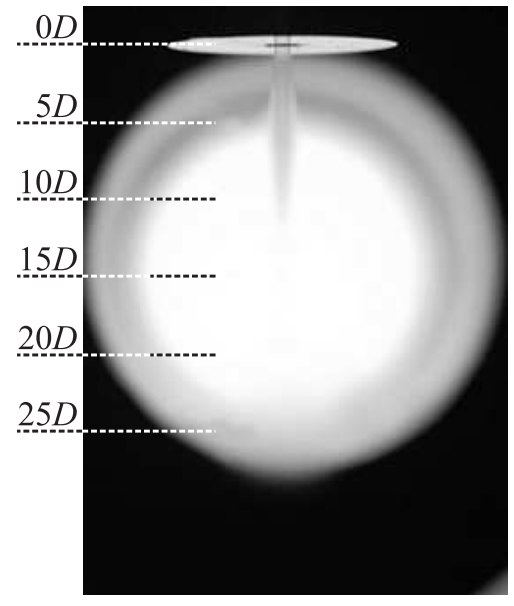

(b)

Figure 6 Time-averaged backlit images of transcritical $(a)$ and supercritical $(b)$ jets

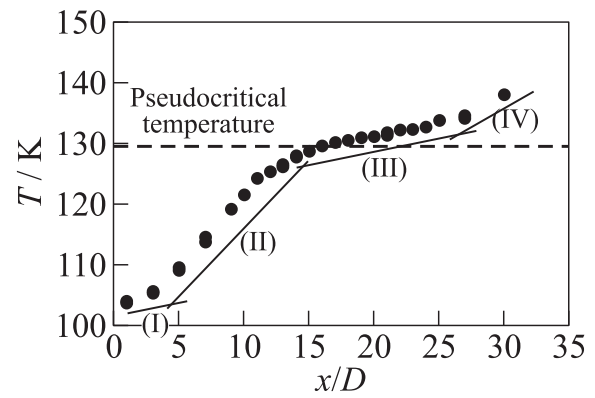

(a)

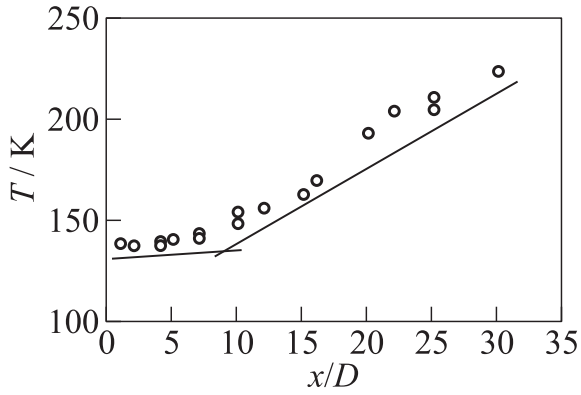

(b)

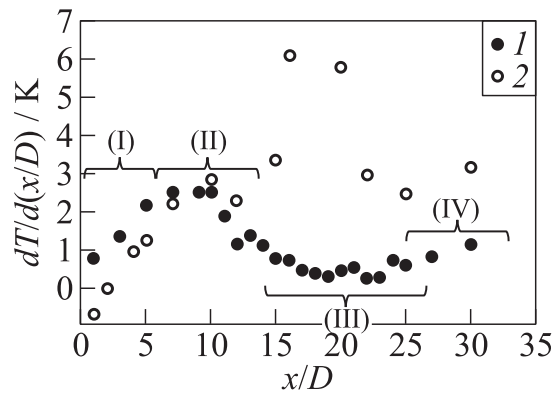

(c)

Figure 7 Temperature profiles of transcritical $(a)$ and supercritical $(b)$ jets and their axial gradient of temperature along the jet centerline $(c): 1-$ transcritical jet; and 2 - supercritical jet 
A decline in the rise of temperature appeared where the local temperature reached near-pseudocritical temperature; hence, it can be considered to be due to the large isobaric specific heat near the pseudocritical temperature as described in Fig. 4c. On the other hand, the temperature profile of the supercritical jet did not have the region with a shallow temperature gradient after the end of the jet potential core. The difference of the temperature gradients of the transcritical and supercritical jets are also observed in Fig. $7 c$.

Figures $8 a$ and $8 b$ plot the axial profiles of the normalized density. The jet density was calculated from the jet temperature at the constant pressure of 4.0 $\mathrm{MPa}$ because both the transcritical and the supercritical jets had sufficiently low Mach numbers to be regarded as incompressible flows. For comparison, the mass fraction profiles were also plotted for an incompressible variabledensity $\mathrm{CO}_{2}$ jet in air as measured by Cheng and Rodi [7]. The density of the supercritical jet decayed in the curve in a similar manner to that of the incompressible variable-density jet downstream of the end of the jet potential core. For the transcritical jet, the density jump appeared at the position where the

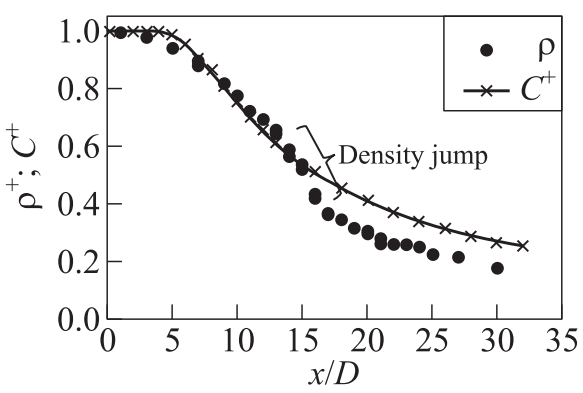

(a)

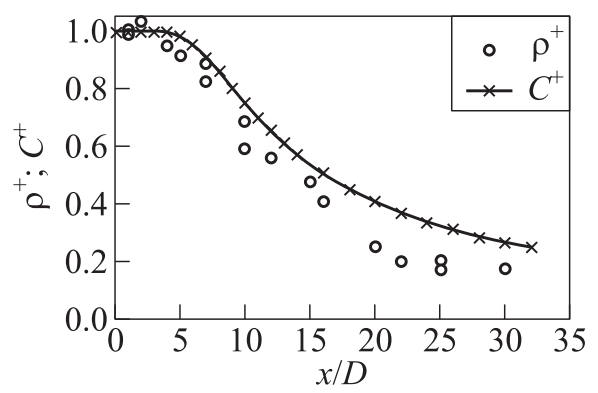

(b)

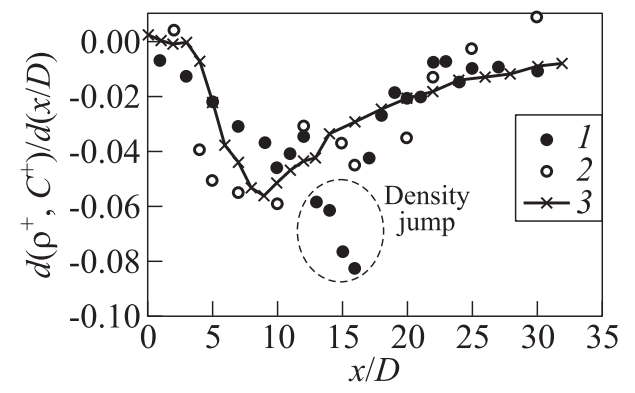

(c)

Figure 8 Normalized density profiles of transcritical $(a)$ and supercritical $(b)$ jets and their axial gradient of density and mass fraction along the jet centerline $(1-$ transcritical jet; and 2 - supercritical jet) compared with the normalized mass fraction profiles of the incompressible variable-density jet (3) [7] (c) 
local temperature was equal to the pseudocritical temperature. Similar to the unique profile of the jet temperature in this region, it can be correlated to the sudden expansion due to pseudovaporization. To clarify, the axial gradient of the normalized density and mass fraction are plotted in Fig. $8 c$. The gradient of the transcritical jets near $x / D=15$ is significantly lower than those of the supercritical and incompressible variable-density jets.

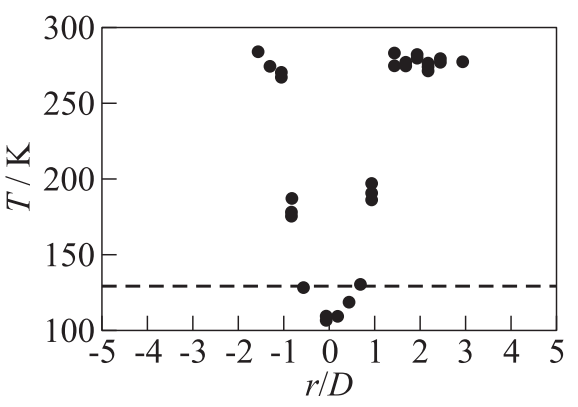

(a)

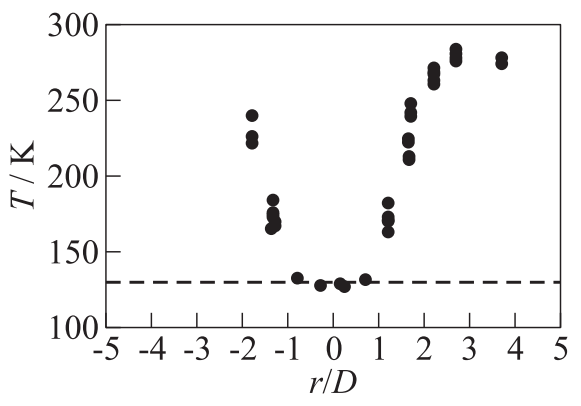

(c)

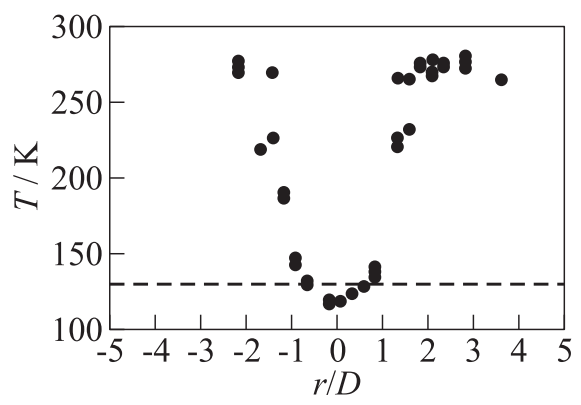

(b)

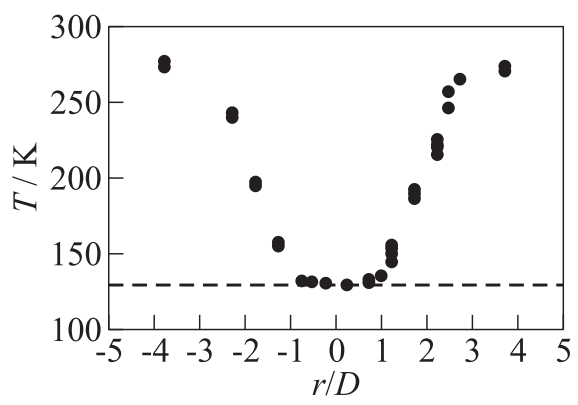

(d)

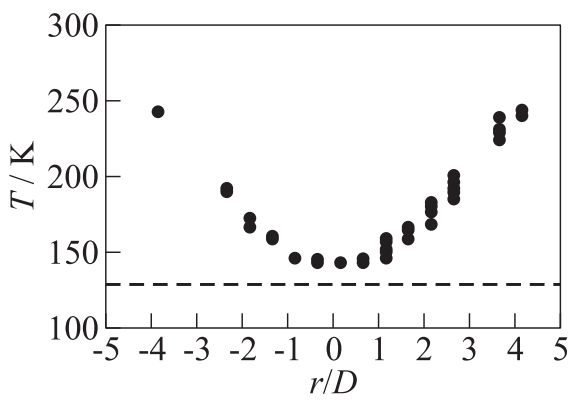

(e)

Figure 9 Radial profiles of temperature at $x / D=5(a), 10(b), 15(c), 20(d)$, and $35(e)$. Dashed line indicates the pseudocritical temperature 


\subsection{Radial Profiles}

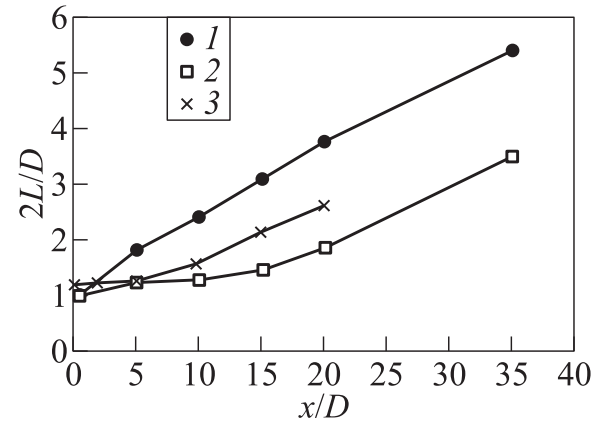

Figure 10 Evolution of FWHM of temperature and density compared with experimental data of the incompressible variable-density jet [7]: 1 - transcritical jet $\left(T^{+}\right) ; 2-$ supercritical jet $\left(\rho^{+}\right)$; and 3 - variable-density jet $\left(C^{+}\right)$
Figure 9 shows the radial profiles of the transcritical jet temperature several axial distances from the injector exit. In the radial profiles at the positions where the jet centerline temperature did not reach the pseudocritical temperature (at $x / D<20$ ), the temperature gradients in the radial direction in the region where the local temperature was still lower than the pseudocritical temperature (at $|r / D|<1$ ) are shallower than those at $|r / D|>1$. Hence, the influence of pseudovaporization also appeared in the radial profiles of the temperature. The temperature gradients at $|r / D|$ $<1$ become small as the mixing

layer develops. Once the jet centerline temperature exceeded the pseudocritical temperature (at $x / D=35$ ), the decrease of the rise in temperature was no longer observed and the profile became similar to a Gaussian distribution.

Figure 10 shows the axial evolution of the full width at half maximum (FWHM) of the radial temperature and density profiles. The FWHM of density was determined by the radial profiles of the nondimensional density as calculated from the temperature at a constant pressure of 4.0 MPa. The FWHM of density stayed almost constant further downstream of the end of the jet potential core $(0<x / D<15)$, whereas that of temperature immediately increased downstream of the injector exit. Note that the FWHM of the mass fraction profiles of the incompressible variable-density $\mathrm{CO}_{2}$ jet began to increase near the end of the jet potential core. Hence, the evolutions of the jet mixing layer of transcritical jets and variable-density jets can be considered to differ owing to pseudovaporization.

\section{CONCLUDING REMARKS}

The temperatures of transcritical and supercritical nitrogen jets were measured to explore the influence of pseudovaporization upon jet mixing.

Along the jet centerline, the decline in the temperature rise at the position where the local temperature reached near-pseudocritical temperature was caused by the large peak of isobaric specific heat. Furthermore, the density jump appeared near the pseudocritical temperature; this can be correlated to the sudden 
expansion due to pseudovaporization. On the other hand, the axial profiles of the temperature and density of the supercritical jet monotonically increased and decreased, respectively, in the downstream region of the end of the jet potential core.

Similar to the axial profiles, the radial profiles of the temperature of the transcritical jet were influenced by pseudovaporization, i. e., the temperature rise in the radial direction became shallow in the region where the local temperature was lower than the pseudocritical temperature. The FWHM of the density profiles stayed almost constant further downstream of the end of the jet potential core, whereas that of the mass fraction profiles of the incompressible variable-density jet began to increase near the end of the potential core. Hence, the evolutions of the jet mixing layers of transcritical jets and incompressible variable-density jets can be considered to differ due to pseudovaporization.

\section{REFERENCES}

1. Oschwald, M., J. J. Smith, R. Branam, J. Hussong, A. Schik, B. Chehroudi, and D. Talley. 2006. Injection of fluids into supercritical environments. Combust. Sci. Technol. 178:49-100.

2. Kim, T., Y. Kim, and S. Kim. 2011. Real-fluid flamelet modeling for gaseous hydrogen/cryogenic liquid oxygen jet flames at supercritical pressure. J. Supercrit. Fluid $58(2): 254-262$.

3. Zong, N., and V. Yang. 2006. Cryogenic fluid jets and mixing layers in transcritical and supercritical environments. Combust. Sci. Technol. 178:193-227.

4. Hosangadi, A., C. P. Lee, C. Kannepalli, and S. Arunajatesan. 2008. Threedimensional hybrid RANS/LES simulations of a supercritical liquid nitrogen jet. AIAA Paper No. 2008-5227.

5. Terashima, H., S. Kawai, and N. Yamanishi. 2011. Investigations of trans/supercritical jet mixing using a high-resolution numerical method. AIAA Paper No. 2011-3935.

6. Jarczyk, M., and M. Pfitzner. 2012. Large eddy simulation of supercritical nitrogen jets. AIAA Paper No. 2012-1270.

7. Cheng, C. J., and W. Rodi. 1980. Vertical turbulent buoyant jets - a review of experimental data. The science and application of heat and mass transfer. New York, NY: Pergamon Press. 83 p.

8. Branam, R., and W. Mayer. 2003. Characterization of cryogenic injection at supercritical pressure. J. Propul. Power 19(3):342-355.

9. Metay, B., E. Robert, R. Viladrosa, C. Cachoncinlle, M. J. Pouvesle, W. Mayer, and G. Schneider. 2003. X-ray diagnostics of the near injector zone of cryogenic nitrogen jets at supercritical pressures. 25th Congress (International) on High-Speed Photography and Photonics. 